\title{
Rol de los profesionales de enfermería en el sistema de salud colombiano
}

\author{
Role of nursing professionals in the Colombian health system
}

Oneys del Carmen De Arco-Canoles ${ }^{*}$ orcid.org/0000-0003-2915-3441

Zuleima Karina Suarez-Calle ${ }^{1}$ orcid.org/0000-0003-2449-8811

1 Facultad de Enfermería, Universidad Antonio Nariño. Bogotá, Colombia.

\section{Resumen}

Introducción: El profesional de enfermería es capaz de brindar cuidados a personas y comunidades, desde los diferentes roles que asume en el sistema de salud y que inciden en la calidad de vida de la sociedad. Objetivo: identificar en la evidencia científica publicada entre 2011 y 2017 el rol de enfermería en el sistema de salud. Materiales y Métodos: La búsqueda bibliográfica se realizó en las bases de datos SciELO, PubMed, LILACS y ScienceDirect, se seleccionaron artículos completos, en español e inglés que presentaron en el título los descriptores: enfermería, rol de enfermería, sistemas de salud, practica avanzada del profesional. Resultados: Se seleccionaron cincuenta estudios publicados entre los años 2011 a 2017, que estudiaron los sistemas de salud en Latinoamérica, buscando orientar el rol de enfermería; resultando tres categorías de interés: prácticas en el ámbito hospitalario, prácticas en el ámbito comunitario y la gestión docencia e investigación. Conclusión: A pesar de la importancia del rol que desempeñan los profesionales de enfermería en Colombia, no se observa una diferenciación de perfiles y funciones dentro del equipo sanitario, por tanto, es preciso delimitar algunas funciones, recuperar campos de acción, afianzar el liderazgo, la autonomía y la humanización en la prestación de los servicios.

Palabras clave: Rol de la enfermera; sistemas de salud; práctica profesional. (Fuente: DeCS, Bireme).

\begin{abstract}
Introduction: The nursing professional is able to provide care to people and communities from the different roles he or she assumes in the health system and that affect the quality of life of society. Objective: To identify in the scientific evidence published between 2011 and 2017 the role of nursing in the health system. Materials and methods: The bibliographic search was carried out in the data bases SciELO, PubMed, LILACS and ScienceDirect. Complete articles were selected in Spanish and English that presented in their titles the descriptors: nursing, role of nursing, health systems, advanced professional practice. Results: Fifty published studies were selected, which studied the health systems in Latin America, seeking to orient the role of nursing between 2011 and 2017. Three categories of interest could be found: Internships in the field of hospitality, community-based practices and teaching and research management. Conclusion: Despite the importance of the role played by nursing professionals in Colombia, there is no differentiation of profiles and functions within the health team; therefore, it is necessary to delimit some functions, to recover fields of action, strengthen leadership, autonomy and humanization in the provision of services.
\end{abstract}

Keywords: Nurse's role; health systems; professional practice. (Source: DeCS, Bireme).

*Autor de correspondencia Oneys del Carmen De Arco Canoles e-mail: dearco.canoles@uan.edu.co dearco.canoles.oneys@gmail.com 


\section{Introducción}

La enfermería es una disciplina profesional que abarca cuidados autónomos y en colaboración que se ofrecen a las personas, familias y grupos poblacionales, enfermos o sanos; estos cuidados incluyen la promoción de la salud, la Prevención de la enfermedad y los cuidados de las personas en condición de discapacidad e incluso moribundas(1). Según el Consejo Internacional de Enfermería (CIE), las funciones esenciales del profesional son: la defensa, el fomento de un entorno seguro, la investigación, la participación en la política de salud y en la gestión de los pacientes y los sistemas de salud, y la formación(1). Deben ir anclados al uso de las teorías y modelos propios de la disciplina que se han generado para impactar de manera positiva sobre la vida de los pacientes y de sus cuidadores directos e indirectos, primarios y/o secundarios.

El profesional de enfermería debe estar en capacidad de brindar cuidados a todas las personas, independientemente de su condición; esta atención debe garantizar el bienestar y la seguridad de las personas, preservando su salud, que se define por la Organización Mundial de la Salud (OMS) como "el estado de completo bienestar físico, mental y social y no solamente la ausencia de afecciones o enfermedades". Para lograr su objetivo debe estar en permanente desarrollo y fortalecer actitudes y valores que permitan la humanización en su quehacer cotidiano, siendo reflexivo, crítico, comprometido, humanista, solidario, respetuoso, honesto, creativo, participativo y responsable para atender al individuo, en un sistema de salud que busca satisfacer las necesidades de la población a cuidar. La enfermería se ha identificado como una profesión humanista, centrada en el cuidado individual, colectivo y de entornos, a partir del desarrollo de sus acciones $^{(2)}$.

Debido a la complejidad de alguna de las acciones desarrolladas por enfermería, Soto et al.(3), plantean que estos cuidadores manejen competencias, entendidas como el conjunto de comportamientos que incluye conocimientos, habilidades y atributos personales que son fundamentales para el logro de un trabajo exitoso; de acuerdo a sus funciones y los estándares requeridos en el empleo, dentro de las cuales se deben considerar: el conocimiento, la comprensión y el juicio; las habilidades cognitivas, técnicas $\mathrm{o}$ psicomotoras $\mathrm{e}$ interpersonales y las características y actitudes personales, que permitirán el desarrollo de las actividades en el ámbito hospitalario, comunitario o combinando desde los escenarios de docencia e investigación $(3,4)$.

Estos elementos permiten desarrollar de manera óptima el arte del cuidado, expuesto por Lopera, et al.(5), como un concepto multidimensional en permanente construcción, que como lo comenta De la Rosa y Zamora(6), ha sufrido una invisibilización, debido a la sobrecarga laboral, la escasa infraestructura institucional, la falta de recursos materiales para la atención y la cantidad de personas que se deben atender en un servicio; no obstante el cuidado es la identidad del profesional de enfermería y cuando estos no pueden cumplir con su tarea de manera adecuada, sienten que se les está limitando su autonomía y su capacidad de acción.

El CIE, resalta la importancia de diferenciar las funciones de los profesionales de enfermería, con las de otros profesionales sanitarios y reconoce que deben hacerse distinciones entre las categorías del personal según sus conocimientos, funciones, contexto laboral, responsabilidades y la formación académica, para establecer y aplicar normas aceptables de práctica clínica, gestión, investigación y formación de enfermería, que permitan cumplir con los cuatro deberes fundamentales por ellos establecidos, así: promover la salud, prevenir la enfermedad, restaurar la salud y aliviar el sufrimiento, de manera tal que se dignifique la vida, se humanice la prestación de los servicios y se ofrezca un cuidado de calidad(7).

Todo este desarrollo de tareas, funciones y actividades ofrecidos por los profesionales de enfermería se realizan dentro de un sistema de salud, que la OMS, define como la suma de todas las organizaciones, instituciones, recursos y personas, cuya finalidad primordial es mejorar la 
salud de la población, que debe prestar servicios que respondan a las necesidades de la población y sean equitativos desde el punto de vista financiero, al tiempo que se ofrezca un trato digno a los usuarios( ${ }^{(8)}$.

Este sistema de salud requiere personal, fondos, información, suministros, transporte, comunicaciones, orientación y direcciones generales. A su vez este talento humano, constituye un elemento fundamental en la producción de los servicios de salud(9); su contribución a la ejecución efectiva de políticas de salud y a la estructuración de sistemas de salud y de servicios adecuados es ampliamente reconocida como esencial(10-13).

Lorenzinini, et al.(14), mencionan que para que un sistema de salud se consolide, debe tener tres elementos que la Organización Panamericana de la Salud (OPS)(15), define como: la población y el territorio, la estructura logística y los modelos de atención y gestión, cuando un sistema se desarrolla con estos tres elementos, su función se orienta a determinar la estructura y el funcionamiento de las instituciones prestadoras de servicios de salud y la seguridad social, por medio de la reglamentación de aspectos relacionados con la calidad de la atención, los mecanismos para la accesibilidad a los servicios de salud, los sistemas de información, los deberes y derechos de cada uno de los actores involucrados, guías y protocolo de atención y los planes de beneficios, entre otros más(16-18).

En Colombia, la ley estatutaria 1751 del 2015(19), busca garantizar el derecho fundamental a la salud, entendiéndolo como autónomo e irrenunciable en lo individual y en lo colectivo; y donde el Estado será responsable de asegurar la igualdad de trato y oportunidades en el acceso a los servicios de salud. A su vez en el artículo 49 de la Constitución Política de Colombia de $1991^{(20)}$, se establece que la atención de la salud es un servicio público a cargo del Estado, y se debe garantizar a todas las personas el acceso a los servicios de promoción, protección y recuperación de la salud. Esta ley surge como respuesta a las limitaciones evidenciadas en el Sistema General de Seguridad Social en Salud, buscando dar solución a la problemática que existe en Colombia porque la población no podía acceder a los servicios de salud(21).

Atendiendo las características anteriores de la enfermería, sus funciones y el sistema de salud, es preciso analizar el rol que vienen desempeñando los profesionales de enfermería dentro del sistema de salud colombiano y el marco legal, que busca garantizar el derecho a la vida y a la salud, con cuidadores capacitados para ejercer su labor orientados a una persona, a colectivos y al entorno(10,22). Los cuidadores en salud, históricamente, reconocidos como profesionales de enfermería, en ocasiones se alejan de la labor del cuidado y cumplen funciones administrativas asignadas como prioridad, lo que contribuye a la práctica invisible del rol de los mismos, y limita o no permite ver de manera clara el rol de un profesional con vocación y servicio humanizado orientado a garantizar y mejorar la calidad de vida de las personas cuidadas ${ }^{(4)}$.

Durante el análisis de la revisión bibliográfica se encontró una separación entre la práctica y el conocimiento adquirido en las instituciones académicas, con la realidad del profesional de enfermería, donde la práctica está alejada del liderazgo y la autonomía que se espera en cada profesional. Por tanto, el interés de esta revisión bibliográfica es responder a la interrogante que surge del análisis: ¿Cuál es el rol que tiene los profesionales de enfermería en el sistema de salud colombiano?.

\section{Materiales y métodos}

La búsqueda se realizó en las bases datos bibliográficas SciELO, PubMed, LILACS y ScienceDirect, se seleccionaron artículos completos, en los idiomas español e inglés, publicados entre 2011 y 2017 que presentaron en el titulo los descriptores: "enfermería" "rol de enfermería" "sistemas de salud" "practica avanzada del profesional". Se descartaron aquellos estudios que, a pesar de referirse al tema del rol de enfermería, se relacionaban básicamente con síndromes o patologías 
específicas que se centraban en la patología y no en el rol del profesional.

La Tabla 1 muestra las bases de datos bibliográficas revisadas, la cantidad de artículos encontrados y los artículos seleccionados para la revisión bibliográfica. Se incluyeron 50 artículos que cumplían los criterios mencionados previamente.

Tabla 1. Bases de datos bibliográficas, y artículos que cumplen con criterios de inclusión.

\begin{tabular}{lcc}
\hline base de datos & $\begin{array}{c}\text { artículos } \\
\text { encontrados }\end{array}$ & $\begin{array}{c}\text { artículos } \\
\text { seleccionados }\end{array}$ \\
\hline SciELO & 10.700 & 35 \\
PubMed & 555 & 9 \\
ScienceDirect & 336 & 5 \\
LILACS & 818 & 1 \\
\hline
\end{tabular}

Se elaboró una base de datos en Excel que permitió obtener información relevante para ésta revisión, donde se incluyeron elementos como: autor (es), título, resumen, metodología, año publicación, país donde se realizó la investigación, entre otros.

Posteriormente se realizó un resumen estructural de cada uno de los artículos, se analizó el tipo de investigación de acuerdo con el rol que desempeña el profesional de enfermería en los sistemas de salud y se apoyó esta revisión con la bibliografía de los entes reguladores de la práctica de enfermería en Colombia.

\section{Resultados}

Rol del profesional de enfermería en el ámbito hospitalario: El cuidado que ofrece el profesional de enfermería se centra en la persona y su interacción con el entorno, el objetivo del profesional debe ser esencialmente, para el beneficio de la persona, hecho que conlleva a que su trabajo sea centrado en ella, su familia y la comunidad.

Dentro de las actividades asistenciales, autores como Seguel, et al.(6), Hanna y Villadiego(23), destacan la valoración física, remisión y control de síntomas, admisión de pacientes al servicio, administración de tratamientos, orientación a los familiares acerca de la salud de los pacientes, cuidados relacionados con la higiene $\mathrm{y}$ alimentación, preparación del paciente para intervenciones, actualización de historias clínicas, revisión de ordenes médicas, vigilancia epidemiológica, acciones de rehabilitación, actividades de promoción de la salud y prevención de la enfermedad, todas estas actividades requieren de conocimientos científicos y de habilidad práctica para garantizar un buen procedimiento y de acuerdo a los protocolos institucionales, algunos son de carácter único del profesional de enfermería. Adicionalmente, se encuentra la dirección de servicios de enfermería y de unidades médicas donde se cuenta con profesionales interdisciplinarios.

Para el desarrollo asertivo del rol, el profesional debe poseer distintas capacidades en las que se resaltan:

Autonomía: Es un concepto de interés para los profesionales clínicos y para los administradores de enfermería y otros líderes. Tal como lo define Guerrero-Núñez y Cid-Henríquez en esta revisión se entenderá como autonomía, la capacidad de tomar decisiones y ejecutar actos de cuidado, donde el profesional de enfermería no requiere la supervisión de otro profesional del equipo interdisciplinario, para cumplir con su labor (24).

La enfermera fundamentará su actuar con el conocimiento de su disciplina, y también se apoyará en otras que permitan un abordaje integral de la persona, la familia, el grupo o la comunidad receptora de dicho cuidado. La autonomía se relaciona entonces con la posibilidad de aplicar conocimientos generados a través de la investigación en la práctica para cambiar los cuidados de atención a las personas, familia y comunidad, según la mejor evidencia disponible y hacerse responsable de ello(25).

En relación a la autonomía del profesional de enfermería se han dado diferentes definiciones, en las que se incluyen:

- La regulación de la conducta por normas que surge del propio individuo. Autónomo es 
todo aquel que decide conscientemente que reglas son las que van a guiar su comportamiento(26).

- La modernidad trajo consigo la conceptualización de las personas como seres intelectuales y moralmente autónomos, con capacidad de raciocinio, juicio y decisión, y con capacidad para decidir sus propias escalas de valores y sus propias ideas del bien y de la felicidad(27).

Dentro de esta revisión se resalta, la definición que plantea la autonomía como la libertad para actuar en lo que se sabe, puesto que un profesional tiene un conocimiento científico, holístico, y un conocimiento general de los campos de acción de la profesión; sin embargo reconociendo los intereses de cada profesional, generalmente se consolida su formación posterior y su experticia en un campo específico. Como profesional de salud, está en toda la capacidad de concertar del cuidado de la persona a partir de los argumentos científicos, donde prevalezca el respeto, los valores y el bienestar de la persona cuidada.

Liderazgo: Se refiere a estar apto a asumir posición de líder en el equipo profesional de salud, objetivando el bienestar de la comunidad, y considerando en su ejercicio el compromiso, la responsabilidad, la empatía, la habilidad para toma de decisiones, la comunicación y la administración de forma efectiva y eficaz(28).

Es importante y relevante el liderazgo en los profesionales de enfermería, porque son estos los que proponen y ejecutan los planes de cuidado y son capaces de involucrar y guiar a sus pares en el proceso de atención. No obstante, la realidad es que a pesar de que enfermería influye y modifica conductas en el paciente y su entorno, este proceso de liderazgo no siempre es valorado por la sociedad y por el propio equipo interdisciplinario de salud(29).

En las instituciones exigen al profesional de enfermería tener una adecuada formación sobre gestión, administración y un desarrollo de habilidades personales e interpersonales que facilitan el logro de la misión y visión de la organización; esto justifica que el profesional de enfermería sea ese líder que permite la participación y planeación de actividades en la práctica profesional dentro de un equipo multidisciplinario(30). El profesional requiere de un liderazgo que le permita participar en la toma de decisiones, a generar nuevas ideas y ayudar en la solución de problemas que presentan las personas, la familia y la comunidad.

Cuidado: Es el objeto de estudio de los profesionales de enfermería y es una necesidad que permite el desarrollo, mantenimiento y conservación de la salud del individuo, su familia y la comunidad. El cuidado de enfermería se distingue del cuidado innato de los seres humanos, porque éste trata de establecer un proceso intencional de querer ayudar a los otros en sus procesos de salud y enfermedad, muerte y renacimiento, fundando y guiando este cuidado en el conocimiento teórico y científico(31).

García(32), afirma que "el cuidado de enfermería es un acto que implica el abordaje integral de la persona, el tratar de establecer límites que impiden apreciar la riqueza del acto mismo del cuidar". Chuaqui, et al.(33), por su parte mencionan que la gestión del cuidado es percibida como un proceso positivo para la enfermería, y está relacionada con lograr mayores niveles de formalización de los procesos administrativos y clínicos de la disciplina.

Resulta pertinente usar el concepto de García, que hace referencia al abordaje integral de la persona, puesto que el profesional de enfermería proporciona cuidados holísticos en entornos sanos y patológicos; en el primero buscando preservar y mantener la salud y en el último desde el inicio del evento hasta su desenlace. Este cuidado se ofrece gracias a los conocimientos adquiridos durante la formación académica, para que el paciente recupere su salud(34).

Los profesionales de enfermería son considerados agentes de cambio para el cuidado de la salud, y se considera que técnicas de enfermería como: tejer alianzas con miembros de la comunidad y cambios de atención de la salud, 
son prácticas de enfermería; estos nuevos roles surgieron como respuesta a los problemas de salud de las personas y de las políticas sociales de salud externas(35), permitiendo reconocer el contexto en el cual se brinda el cuidado y las características propias de la comunidad.

El profesional está en todos los lugares, lidera los diferentes procesos de cuidado y tiene en cuenta las diversas dimensiones y relaciones humanas. Sin embargo, frecuentemente tiene dificultad de organizarse por la falta de autonomía y liderazgo en el objeto de su trabajo(36), sin embargo desde su experticia se puede ubicar en áreas de cuidado crítico, urgencias adultos y pediátricas, ginecoobstetricia, medicina interna, salas de cirugía, recuperación o quirúrgicos, ortopedia, pediatría, unidades de cuidados intensivos pediátricas y adultos, sala de partos, lactancia materna, unidad de quemados, salud mental, entre otras, y en todas sus labor se diferencia de los otros profesionales porque se centra en el cuidado de la persona sana o enferma.

Rol del profesional de enfermería en el ámbito comunitario o territorial: En este ámbito, se busca aplicar los conocimientos en salud pública y las habilidades prácticas de enfermería como estrategias en la comunidad con el fin de promover, mantener y restaurar la salud de la población.

En Colombia la profesión de enfermería se reglamenta con la Ley 266 de 1996(37), y la Ley 911 de 2004(38), las cuales exponen los parámetros de las funciones de la profesión; algunas funciones contribuyen de forma específica a que los individuos, la familia y la comunidad adquieran habilidades, hábitos y conductas que fomenten su autocuidado, en la promoción de la salud y la prevención de la enfermedad.

Dentro de la Resolución 0429 de $2016^{(39)}$ por medio de la cual se adopta la Política de Atención Integral en Salud, se define el modelo integral de atención en salud (MIAS), que enuncia a su vez los componentes propios del modelo, donde el profesional de enfermería tiene la posibilidad y la responsabilidad de cumplir con sus funciones como: integrar los conocimientos teóricos y las habilidades prácticas con la salud pública; poseer determinadas habilidades sociales, actitudes y convicciones personales; ofrecer sus servicios a la totalidad de la población sin distinción de raza, credo y/o condición social; la actuación se fundamenta en las necesidades y los problemas de salud prioritarios de la población(40).

Entre este ámbito se encuentra la formulación y el desarrollo de los Planes de Salud Territoriales (PST), regulados a través de la Resolución 1841 de 2013(41), donde establece la dirección de acciones individuales y colectivas en el orden departamental, distrital y municipal según el Plan Decenal De Salud Pública 2012-2021. Por lo tanto, los profesionales de enfermería se pueden desempeñar como coordinadores de los PST, líderes del proceso administrativo y/o gerentes en el área de salud pública, en donde diseñan, ejecutan y evalúan las acciones de salud en mejora del bienestar y la calidad de vida de la población.

Otro escenario en el que los profesionales con enfoque comunitario pueden desempeñarse es el territorio, algunos programas en Bogotá han demostrado que existe un impacto positivo, cuando el profesional de enfermería llega al territorio y se ofrece la atención en un sitio conocido y familiar, tal como la casa, la escuela o el lugar de trabajo(53).

Los servicios de enfermería basados en la comunidad, presentan una gran oportunidad para que los profesionales de enfermería mejoren sus contribuciones a la cobertura universal de salud; donde el profesional, tendrá que abogar por un modelo de prestaciones de servicio en salud, direccionado en la mejora de la cobertura de atención, así como la ampliación del alcance y la profundidad de los servicios de enfermería de la comunidad(42).

Dentro de los modelos de enfermería, es preciso mencionar a Nola J. Pender, quien es reconocida por su aporte a través del modelo de promoción de la salud, en el cual planteó que promover un estado óptimo de salud era el objetivo que debía señalar las acciones preventivas del profesional 
de enfermería(38); Esto es relevante en este ámbito, por cuanto en el territorio usualmente se encuentra población sana y a través de este modelo se puede fortalecer la salud, preservarla y mantenerla.

\section{Rol de profesional de enfermería, una mirada} desde la docencia y la investigación: La educación es un componente esencial en el rol del profesional de enfermería, ésta es concebida como una función sustantiva dentro del rol, por tal motivo se consideran que este profesional es educador por naturaleza(43). La educación que brinda el profesional de enfermería no solo es un elemento central del cuidado, sino también de su gestión y transversal al rol que desempeñe en las diferentes instancias de sus responsabilidades, que requiere la innovación constante y estar a la vanguardia en temas de interés y relevantes que den respuesta a las necesidades de la población.

La función docente del profesional de enfermería se refiere principalmente a las actividades de educación para la salud y las relacionadas con la educación continua o formación de los nuevos profesionales, capaces de generar cambios integrales a la sociedad y al ámbito sanitario. Para ejecutar la función, los profesionales de enfermería deben cumplir con requisitos académicos como habilidades propias de la docencia. Al ser coherente que del desarrollo de su labor pueden incentivar al estudiante a la toma de decisiones, a hacer observaciones, a percibir relaciones y a trabajar en equipo. De tal manera el docente, proporciona al estudiante el desarrollo de habilidades y actitudes que conducen a la adquisición de un poder técnico científico, con el fin de poder actuar en beneficio de la $\operatorname{sociedad}(44)$.

La calidad de la docencia del profesional de enfermería debe garantizar la preparación de los futuros profesionales competentes que se identifiquen con su quehacer y que estén alertas a reaccionar al medio y las exigencias de los servicios de salud. Enfermería actualmente ha encontrado en sus quehaceres, oportunidades que la han posicionado como una profesión con bases científicas sólidas, siendo la investigación una herramienta indispensable para el progreso de estos. Es decir, que se debe involucrar desde los primeros semestres a los nuevos profesionales en las investigaciones, buscando despertar su interés temprano.

Los desafíos de la profesión de enfermería plantean la necesidad de continuar un proceso formativo sustentado en la idoneidad, excelencia y calidad, que permitan al profesional situarse y recuperar la autonomía y liderazgo dentro de las disciplinas que brindan el cuidado para la salud. Este proceso obliga a crear modelos y teorías propias que fortalezcan el posicionamiento de la profesión, y desarrollar investigaciones que incrementen el saber del propio campo disciplinar. De este modo, la enfermería para alcanzar o mejorar el estatus social debe seguir ofreciendo conocimiento sobre el objeto de estudio, bases científicas que la sustenten, teorías y modelos que integren las bases del conocimiento, y un número considerable de investigaciones del fenómeno de estudio realizadas por los miembros de la comunidad(45).

La investigación en enfermería es fundamental para desplegar, evaluar y propagar el conocimiento de la profesión. La vinculación del profesional de enfermería en el ámbito de la investigación, se ha hecho con el fin de generar la práctica de enfermería basada en la evidencia; más aún, se motiva a que los profesionales de enfermería a través de las investigaciones propias de su disciplina pueda generar nuevos conocimientos que permitan al profesional evaluar su práctica a través de los costos derivados del cuidado y los beneficios otorgados a los pacientes, garantizando un cuidado costoefectivo de enfermería de calidad(46).

La investigación en enfermería es la fuente de desarrollo del conocimiento, que le da la característica de la disciplina, que ha de estar presente tanto en la práctica clínica como comunitaria y en el desarrollo de la administración y la educación. La sociedad de Honor de Enfermería, Sigma Theta Tau International Honor Society of Nursing(47), fue la primera organización en Estados Unidos que dio fondos para la investigación en enfermería en 1936. Hoy en día, esta organización internacional 
proporciona financiación para la investigación de los profesionales, a través de la recaudación de fondos y la gestión activa.

La investigación se desarrolló de forma fidedigna analizando de manera crítica las historias relacionadas con la profesión, considerándolo un reto a ser enfrentado con profesionalismo, ya que a medida que se conoce la historia de la enfermería, se percibe su vinculación con otras actividades del ejercicio de la salud, así como su participación social(48).

Es posible destacar que la actividad de docencia del profesional de enfermería está diseñada sistemáticamente, donde se constituye un rol motivador y de guía, que orienta a la nueva generación para el crecimiento personal, en el desarrollo académico, estimula la práctica, la capacitación y la actualización de los conocimientos, con recursos de aprendizaje atractivos a través de la ceración de medios acordes a los requerimientos de la población y del sistema educativo.

\section{Discusión}

El fenómeno central de la enfermería es el cuidado, sin embargo, este debería ir de la mano con la ciencia y la práctica, para no generar rupturas. El profesional se caracteriza principalmente por la integralidad de la asistencia en salud, y su capacidad para identificar las necesidades y las expectativas del paciente, la familia y la comunidad, sin embargo, Hanna y Villadiego(23), mencionan que la función principal del profesional de enfermería parece estar sufriendo cambios significativos ya que el cuidado ha pasado a un segundo plano; también mencionan que los profesionales de enfermería en muchas ocasiones abandonan sus quehaceres cotidianos por realizar actividades de los otros profesionales de la salud, y deben responder por todas las situaciones ocurridas en el servicio.

A partir del reconocimiento de la problemática que se presenta en el campo del profesional de enfermería en Colombia y a través de la revisión literaria es posible evidenciar que existe un contexto que identifica las relaciones y el desarrollo del profesional y que solo a través de la implementación de políticas incluyentes e integrales será posible alcanzar un rol profesional deseado.

El ámbito asistencial del profesional de enfermería incluye aquellas actividades que preservan la dignidad de la persona, la familia y la comunidad, en donde el cuidado implica el uso de los conocimientos y la sensibilidad respecto a lo que es importante. El objetivo principal de este ámbito es entender lo que es importante, el cual debe ser dirigido al usuario como un ser humano integral, lo que significa que debe ser personalizado $(6,23,32)$.

En el ámbito comunitario se pudo identificar que hay varias áreas en las que los profesionales de enfermería se desarrollan tales como: los planes de salud territoriales, dirección y gestión de la salud pública en escenarios locales, regionales y nacionales, tales como: Secretaria de Salud, el Ministerio de Salud, Congreso, organizaciones de carácter internacional como la OPS, la OMS, entre otras, lo que permite impactar en las políticas y permite generar estrategias y programas de impacto a nivel social, económico y sanitario $(38,40,32)$. Es preciso mencionar que durante las diferentes actividades que realiza el profesional de enfermería se resaltan las capacidades de liderazgo, planeación y organización dentro de los equipos de salud, en el contexto disciplinar y en el contexto inter e intra-disciplinar, tanto a nivel nacional como internacional, donde estos profesionales son los dirigentes de la misma y los proponentes de estrategias que inciden de manera positiva en la población $(23,40,42,43,44)$.

Linares(49), menciona que el liderazgo es la capacidad para manejar el cambio y dar poder a otros para influir en la práctica clínica así como en las políticas sanitarias y en la organización; menciona que los profesionales de enfermería suelen trabajar en compañía de los administradores y liderar proyectos de cambio, creando un entorno saludable de trabajo, con habilidades de resolución de conflictos y trabajo en equipo, son activos en la toma de decisiones y facilita la resolución de conflictos éticos; facilitando la intervención del profesional como 
cuidador y generando la integración de los servicios de salud para la persona, orientados y liderados por el profesional de enfermería.

El aprendizaje de los profesionales de enfermería es transformativo e implica desarrollar cualidades de liderazgo, con el propósito de formar agentes de cambio ilustrados. Esta habilidad debe movilizar tanto a las comunidades académicas y a los profesionales, este es un factor crucial para el éxito de los esfuerzos de la reforma en las instituciones académicas, acerca del liderazgo en los futuros profesionales( ${ }^{(50)}$.

El ámbito de gestión docencia e investigación se refiere principalmente a las actividades de educación para la salud y las relacionadas con la educación continua o formación de los nuevos profesionales. Se evidenciaron las ventajas que este ámbito puede tener en la profesión cuando se usan los modelos y las teorías de la disciplina y los conocimientos prácticos que de ellas se derivan, así como las acciones que permiten contribuir al desarrollo del ejercicio profesional. En este papel el educador no solo educa, sino que también es educado, es decir, ocurre una inversión de roles como consecuencia de la interacción con los estudiantes lo que le permite conocer acerca de la perspectiva de estos, para saber reaccionar y desarrollar una conducta personal $y$ un método que le posibilite proporcionar conocimiento holístico basado en la educación para la salud(51).

El papel de los profesionales de enfermería debe quedar claro tanto para ellos como para las instituciones de salud. La descripción del trabajo debe hacer hincapié en las 5 principales funciones del profesional de enfermería, que incluyen: la atención directa basada en la evidencia científica, la enseñanza y el asesoramiento, la investigación, el liderazgo/gestión y la toma de decisiones éticas. El desarrollo del liderazgo en enfermería es importante, porque los profesionales líderes e investigadores necesitan proporcionar que pueden hacer una diferencia en el sistema de salud(52).
Se deben realizar más revisiones como ésta, para evidenciar los cambios que ha tenido el ámbito laboral de los profesionales de enfermería que son herramientas fundamentales para el cuidado. Las instituciones deben asumir su responsabilidad y los profesionales deberían exigir sus derechos y utilizar las habilidades, los conocimientos adquiridos en la formación académica $\mathrm{y}$ herramientas aprendidas para mejorar sus ámbitos de acción y recuperar ese espacio de interlocución y seguir siendo esos líderes autónomos referentes de cuidado.

\section{Conclusiones}

Pese a la evidencia de la importancia del rol que desempeñan los profesionales de enfermería en beneficio de la persona, la familia y la comunidad, en los sistemas de salud, este no siempre se percibe de manera impactante. Se puede inferir, que en algunas instituciones la situación se presenta, entre otras medidas, para abaratar los costos del sistema de salud y de los cuidados a brindar a las personas, generando sobrecarga laboral por temas administrativos y relegando el objeto de ser de la profesión.

En el ámbito hospitalario, el profesional de enfermería es el responsable del cuidado de la persona, su familia y la comunidad, este no se refiere solamente a suplir la necesidades de ellos sino también involucrar el dominio sobre las situaciones administrativas y asistenciales del servicio que dirige, se evidenció que los profesionales sienten que sus esfuerzos por mantener el funcionamiento de los servicios no se está viendo reflejado, puesto que en ocasiones se sienten invisibles en su quehacer cotidiano, debido a la falta de reconocimiento de sus colegas $\mathrm{y}$ de los pacientes o incluso sus familiares.

En el ámbito comunitario, los profesionales de enfermería tienen campos que pueden aprovechar, en Colombia apoyando en la política PAIS y en el modelo MIAS, sin embargo falta liderazgo, autonomía y la utilización de las estrategias de información para abanderar estos espacios, el profesional debe fortalecer el desarrollo de sus competencias en el quehacer, 
para posibilitar el reconocimiento de la praxis de la profesión y debe apropiarse de los contextos, desde los cuales a través de la historia de la disciplina se ha podido brindar cuidados a personas y poblaciones.

Desde la gestión, docencia e investigación, la formación de nuevos profesionales es una tarea compleja que requiere del desarrollo de competencias no solo relacionadas con sus saberes, sino con habilidades que se adquieren a través de la experiencia, las cuales deben aplicar en tiempo real, es decir en un ambiente complejo como lo son las instituciones de salud; en donde se involucra el sujeto de cuidado, su familia, su entorno, el equipo de salud y otros colegas con los cuales se debe generar una intervención holística.

Es importante destacar que en la actualidad en las instituciones educativas se emplaza a los futuros profesionales de enfermería a desarrollar gestión investigativa y liderazgo para construir entornos de trabajo de calidad, poner en práctica nuevos modelos de atención y contribuir al desarrollo de una fuerza laboral.

Las actividades misionales de la enfermería deben ser posicionadas y diferenciadas de las que realizan otros profesionales de la salud, manteniendo la luz del cuidado como bandera de Enfermería.

Esta revisión posibilitó el reconocimiento del saber y la praxis de la profesión de enfermería que está inmersa en nuevos ámbitos laborales, en los diferentes contextos; se puede afirmar que el rol está relacionado con la promoción de la salud y la prevención de enfermedades, en entornos sanos y patológicos. Por lo tanto, los resultados de esta revisión confirman que el rol de los profesionales de enfermería, contribuyen al mejoramiento en la calidad de vida, la promoción de la salud y la disminución de las enfermedades, para la persona, su familia y la comunidad.

\section{Recomendaciones}

1. El fomento de espacios académicos que permitan el desarrollo de investigaciones relacionadas, en los diferentes campos de acción y así responder a las problemáticas actuales en relación con el rol que se desempeña en las diferentes instituciones.

2. Es conveniente que tanto las instituciones prestadoras de servicios de salud como las instituciones educativas y las agremiaciones profesionales generen estrategias para generar un mejor posicionamiento de esta disciplina histórica y siempre vigente a través del tiempo. Que los profesionales de enfermería participen en el planteamiento de las diferentes acciones interdisciplinarias en los sistemas de salud.

3. Divulgación activa de las investigaciones realizadas por los profesionales de enfermería para mejorar la visibilidad de la profesión y contribuir al desarrollo de nuevos campos laborales que ayuden a posicionar la profesión con más potencialidad.

4. Que los profesionales de enfermería se empoderen, discutan como gremio sus condiciones de trabajo, que se vean como pares de los otros profesionales de la salud, pues su conocimiento también es científico y debe ser respetado y valorado en la comunidad académica y científica, tal como lo han hecho y lo han demostrado personajes como Florence Nightingale, Dorotea Orem, Nola Pender, Virginia Henderson, entre otras destacadas mujeres de esta noble profesión.

\section{Conflicto de interés}

Los autores no refieren conflicto de interés para la realización de esta revisión.

\section{Referencias}

1. Consejo Internacional de Enfermeras. La definición de enfermería. Suiza: CIE; 2015. [cited 2017 Marzo 13]. Available from: http://www.icn.ch/es/who-weare/icn-definition-of-nursing/ 
2. Organización Mundial de la Salud (OMS). ¿Cómo define la OMS la salud?. Ginebra: OMS; 1948. [cited 2017 Marzo $8 . \quad$ Available from: http://www.who.int/suggestions/faq/es/

3. Soto P, Reynaldos K, Martínez D, Jerez O. Competencias para la enfermera/o en el ámbito gestión $\mathrm{y}$ administración: desafíos actuales de la profesión. Aquichan. 2014;14(1):79-99.

4. Seguel F, Valenzuela S, Sanhueza O. El trabajo del profesional de enfermería: revisión de la literatura. Ciencia y enfermería. 2015;(2):11-20.

5. Lopera M, Forero C, Paiva L, Cuartas M. El quehacer cotidiano de la enfermera significa soportar la carga. Cuidarte. 2016;7(1):1260-70.

6. De la Rosa R, Zamora G. Cuidados invisibles: ¿son suficientemente reconocidos? Index Enferme. 2012;21(4):1260-70

7. Consejo Internacional de Enfermería. Código Deontológico del CIE para la profesión de enfermería. Suiza: CIE; 2006. [Cited 2017 Marzo 16]. Available from:

http://publicaciones.san.gva.es/val/prof/enfermeria/d ocumentacion/codigocie2006.pdf.

8. Organización Mundial de la Salud. Acerca de los sistemas de salud. Ginebra: OMS; 2005. [cited 2017 Marzo 28. Available from: http://www.who.int/healthsystems/about/es/

9. García H, Díaz P, Ávila D, Cuzco M. La Reforma del Sector Salud y los recursos humanos en salud. An Fac Med. 2015;76:7-26.

10. Guerrero R, Gallego A, Becerril V, Vásquez J. Sistema de salud de Colombia. Salud Pública de México. 2011;53:144-155.

11. Belló M, Becerril V. Sistema de salud de Argentina. salud pública de méxico. 2011;58:96-108.

12. Gómez O, Sesma S, Becerril V, Knaul F, Arreola H, Frenk J. Sistema de salud de México. Salud Pública de México. 2011;58:220-232.

13. Bonvecchio A, Becerril V, Carriedo Á, Landaeta M. Sistema de salud de Venezuela. Salud Pública de México. 2011;58:275-286.

14. Lorenzini A, Regina de Andrade S, Schaefer A, Crespo L. La atención secundaria en salud: mejores prácticas en la red de servicios. Latino-Am. Enfermagem. 2013;21(Spec):131-139.

15. Organización Panamericana de la Salud. Redes Integradas de Servicios de Salud: Conceptos, opciones de política y Hoja de ruta para su implementación en las Américas. Washington: OPS; 2010. [cited 2017 Abril $15 . \quad$ Available from: http://www.paho.org/uru/index.php?option=com_doc man\&view=download\&category_slug=publicacionessistemas-y-servicios-de-salud\&alias=145-redesintegradas-de-servicios-de-salud-aps-n4\&Itemid=307

16. Rivero E, Amado L. Modelo de salud en Colombia: ¿financiamiento basado en seguridad social o en impuestos? Gerenc. Polit. Salud. 2012;11(23):111-120.

17. Becerril V, Medina G, Aquino R. Sistema de salud de Brasil. Salud Pública de México. 2011;58:120-131.

18. Domínguez E, Zacca E. Sistema de salud de Cuba. Salud Pública de México. 2011;58:168-176.
19. Ministerio de Salud. Ley estatutaria 1751 de 2015, Por la cual se regula el derecho fundamental a la salud y se dictan otras disposiciones. Bogotá: MinSalud; 2015.

20. Corte Constitucional. Constitución Política de Colombia Actualizada con los Actos Legislativos a 2015. Bogotá: Corte Constitucional; 2015.

21. Otero E. Orígenes de las deficiencias del sistema de salud colombiano. Cartagena: El Universal; 2015. [cited 2017 Abril 15. Available from: http://www.eluniversal.com.co/salud/origenes-de-lasdeficiencias-del-sistema-de-salud-colombiano-214184.

22. Becerril V, Reyes JdD, Manuel A. Sistema de salud de Chile. Salud Pública de México. 2011;53:s132-s143.

23. Hanna M, Villadiego M. La administración de enfermería en el área clínica y sus implicaciones en el cuidado. Revista Colombiana de Enfermería. 2014;9:65-70.

24. Guerrero-Núñez S, Cid-Henríquez P. Una reflexión sobre la autonomía y el liderazgo en enfermería. Aquichan. 2015;15(1):129-140. DOI: 10.5294/aqui.2015.15.1.12

25. Luengo C, Paravic T. Autonomía Profesional factor clave para el ejercicio de la enfermería basada en la evidencia. Index de Enfermería. 2016;25(1-2):42-46.

26. Escuelas de Familia moderna. Definición de las competencias. Navarra: Gobierno de Navarra; 2017. [cited 2017 Marzo 28. Available from: https://www.educacion.navarra.es/documents/27590 /51352/AUTONOMIA_Y_RESPONSABILIDAD.pdf/34e7 af0a-341e-47eb-b7a6-5b44a2c56a4e.

27. Arango Bayer G. Enfermería y el respeto a la autonomía en el ámbito hospitalario: entre la sobreprotección y el descuido. av.enferm. 2013;31(1):126-132.

28. Marcellino de Melo G, Schelindwein B. Liderazgo del enfermero: una revisión integradora de la literatura. Latino-Am. Enfermagem. 2011;19(3):1-9.

29. Cárcamo-Fuentes C. y Rivas-Riveros E. Estilo de liderazgo en profesionales de enfermería según su función en los sectores público o privado en Temuco, Chile, 2015. Aquichan. 2017;17(1):70-83. Doi: 10.5294/aqui.2017.17.1.7 Available from: http://www.scielo.org.co/pdf/aqui/v17n1/16575997-aqui-17-01-00073.pdf

30. Bryant D, Valaitis R, Martin R, Donald F, Morán L, Brousseau L. Enfermería con práctica avanzada: una estrategia para lograr la cobertura universal de salud y el acceso universal a la salud. Rev. Latino-Am. Enfermagem. 2017;25:1-11.

31. Contreras V. Evidencia del Arte en Enfermería. Enfermería Global. 2013;(30):236-331.

32. García L. La ética del cuidado y su aplicación en la profesión enfermera. Acta Bioethica. 2015;21(2):311317.

33. Chuaqui J, Bettancourt L, Leal V, Aguirre C. La identidad profesional de la enfermería: un análisis cualitativo de la enfermería en Valparaíso (1933-2010). Aquichan. 2014;14(1):53-66.

34. García MdL, Cárdenas L, Arana B, Monroy A, Hernández Y, Salvador C. Construcción emergente del concepto: cuidado profesional de enfermería. Texto Contexto Enferm, Florianópolis. 2011;20:74-80. 
35. Kamei T, Takahashi K, Omori , Arimori , Hishinuma , Asahara, et al. Por una enfermería de práctica avanzada y una alianza para la atención centrada en las personas, para la Cobertura Universal y Acceso Universal a la salud. Latino-Am. Enfermagem. 2017;25:1-10.

36. Stein, Stein, Biazus C, Lorenzini. Sistema de cuidado de enfermería en la perspectiva luhmanniana. Latino-Am. Enfermagem. 2012;20(5):1-7.

37. El Congreso de Colombia. Ley 266 de 1996: Por la cual se reglamenta la profesión de enfermería en Colombia y se dictan otras disposiciones. Bogotá: MinEducación; 1996.

38. Congreso de Colombia. Ley 911 de 2004, por la cual se dictan disposiciones en materia de responsabilidad deontológica para el ejercicio de la profesión de Enfermería en Colombia; se establece el régimen disciplinario correspondiente y se dictan otras disposiciones. Bogotá: Congreso de Colombia; 2004.

39. Ministerio de Salud y Protección Social. Resolución 0049 de 2016, por medio de la cual se adopta la Política de Atención Integral en Salud. Bogotá: MinSalud; 2016.

40. León P. Percepción del quehacer de enfermería en el Plan de Salud Territorial: una mirada desde enfermería. Inves Enferm. Imagen Desarr. 2014;16(2):13-25.

41. Congreso de Colombia. Resolución Número 1841 de 2013 Por la cual se adopta el Plan Decenal de Salud Pública 2012-2021. Bogotá: Congreso de Colombia; 2013.

42. Yee W, Fung I, Chan E. Cobertura Universal de Salud a través de Servicios Comunitarios de Enfermería: China versus Hong Kong. Rev. Latino-Am. Enfermagem. 2017;25:1-5.

43. Ángel G, López F, Restrepo D. La experiencia educativa del profesional de enfermería en el ámbito clínico. Investig. Enferm. Imagen Desarr. 2013;15(2):9-29.

44. Bettancourt L, Muñoz L, Barbosa M, Fernades M. El docente de enfermería en los campos de práctica clínica: un enfoque fenomenológico. Rev. Latino-Am. Enfermagem. 2011;19(5):1-9.

45. Aguayo M, Castelló M, Monereo C. La identidad del académico de enfermería: entre la docencia y la investigación. Texto Contexto Enferm, Florianópolis. 2014;23(2):241-249.

46. Álvarez D. Enfermería en América Latina: una mirada al horizonte. Av Enferm. 2015;33(2):295-305.

47. Sigma. Sigma Organizational Fact Sheet. Indianápolis: Sigma; 2017. [cited 2017 Abril 15]. Available from: https://www.nursingsociety.org/connectengage/about-stti/sigma-theta-tau-internationalorganizational-fact-sheet

48. Gómez D. Ascenso de enfermeras a la Gerencia Académica: discutiendo su rol según la Teoría de Adaptación de Roy (años 70-80). Revista Brasileira de Enfermagem . 2013;66(5):753-759.

49. Del Barrio-Linares M. Competencias y perfil profesional de la enfermera de práctica avanzada. Enferm Intensiva. 2014;25(2):52-57.

50. Frenk, Chen, Bhutta Z, Cohen J, Crispf, Evans, et al. Profesionales de la salud para el nuevo siglo: transformando la educación para fortalecer los sistemas de salud en un mundo interdependiente. Educ Med. 2015;16(1):9-16.

51. Islas P, Pérez A, Hernández G. Rol de enfermería en educación para la salud de los menonitas desde el interaccionismo simbólico. Enfermería Universitaria. 2015;12(1):28-35.

52. Maaitah R, Fawzi R. Exploración de las acciones prioritarias para el fortalecimiento del papel de las enfermeras en el logro de la cobertura universal de salud. Latino-Am. Enfermagem. 2017; 25.

53. Segura J. Territorios saludables: la joya de la salud comunitaria en Bogotá. Salud pública y otras dudas. 2016 Noviembre: p. 1-84 